\title{
Research on Safety Distance Mathematical Model of Pro-Active Head Restraint in Rear-end Collision Avoidance System
}

\author{
Xiaoqin Yin $^{1}$ and Mingxia Wang ${ }^{1}$ \\ ${ }^{1}$ School of Mechanical Engineering, Jiangsu University, Zhenjiang 212013, China \\ xqyin@mail.ujs.edu.cn,wangmx_1005@163.com
}

\begin{abstract}
Pro-active head restraint is a new automotive safety device with both active safety and passive safety characteristics. It can make a pre-estimation of the occurrence possibility of rear-end collision. Thus, the whiplash injury can be effectively reduced or even prevented. One of the key elements in rear-end collision avoidance system is to establish an effective safety distance mathematical model. Based on the running state of front car, the related calculation models of safety distance are established by means of dynamical and kinematical analysis of vehicle braking process and following process. Taken randomness and dynamic characteristics of parameters into consideration, the fuzzy relations between these parameters should be validated by means of fuzzy theory. Thus, it may accord with the actual driving conditions. By using the MATLAB software, the study shows that safety distance model and the methods used to determine parameters are reasonable, and the false alarm can be effectively minimized.
\end{abstract}

Keywords: Pro-active head restraint; Safety distance; Mathematical model; Fuzzy theory; Rear-end collision avoidance system

\section{Introduction}

With the rapid development of the automobile industry and transportation in China, automotive safety has received widespread attention from the general public. According to the road traffic accident statistics of Japan in 2003, the incidence of rear-end collision accounts for more than $30 \%$ of the traffic accidents, and about $90 \%$ of passengers' injury in rear collision is neck injury which is called whiplash injury [1]. Though the possibility of occupant's fatal injury caused by whiplash injury is relatively small, the collision may lead to occupant's lasting neck pain, and huge social cost for treatment [2]. Therefore, many countries pay attention to the whiplash injury and a new active head restraint was invented to reduce occupant's neck injury in rear impact. Traditional mechanical headrest can only be activated at the time of peak intervertebral rotations and its potential protective effect for reducing whiplash injuries is decreased [3].Researches show that if drivers can take measures in advance by one second, $60 \%$ of rear accidents will be avoided and $30 \%$ of direct economic losses will be reduced [4]. Thus, pro-active head restraint both active safety and passive safety characteristics is designed. It can make a pre-estimation of the occurrence possibility of rear collision, which may reduce or prevent the whiplash injury effectively.

As a prerequisite of automobile rear-end collision preventing system, the accuracy and real-time capability of safety distance mathematical model has direct influence on the reliability of the system, and it can also reduce the false alarm rate [5]. In this paper, a safety distance model, which can be applied in complex and variable traffic environments, is established. Risks of multi-object and all kinds of uncertain factors are determined by fuzzy theory and quantitative analysis is carried out by means of membership functions of fuzzy theory. Finally, the multi-objective automobile anti-collision system is realized by means of the MATLAB simulation software. In addition, the algorithm of this system is simple, and the system is easy to operate. It can process the data and make a reasonable 
response timely and efficiently, which can improve the performance of automotive anticollision system and automotive security.

\section{The Rear-end Collision Avoidance System Inside the Pro-active Head Restraint}

This system is mainly composed of artificial selection unit, signal acquisition unit, processing control unit, display and alarm unit, and implement unit, and each unit contains the respective sub-module. With the distance sensor, this system monitors and displays the distance between two cars timely. Then, it predicts rear collision through the traffic safety distance algorithm. If there exists the risk of rear collision, the system will remind the driver of taking measures to avoid collision by means of screen display and brake lights. If the driver fails to perform any effective operation and the risk of collision continues to rise, the active headrest will be triggered by implement unit to decrease the occupant's neck injury ultimately.

\section{The Establishment of Safety Distance Mathematical Model}

As the automobile auxiliary system, a relatively reasonable safety distance model should be established in this system. Thus, it can make accurate judgments about the target vehicle so that it cannot only guarantee safe driving between cars but also increase the utilization rate of road [6].

\subsection{The Model Assumption of Automotive Driving Condition}

In attempt to develop the reliable mathematical model, some assumptions are made as follows. The front and following cars are defined respectively as $A$ and $B$, which are driving in the same direction and on the same lane. As the first vehicle is a non-following state, this paper just considers the safety distance with the behind. Moreover, due to the complexity and variability of automotive driving conditions, the driver is assumed to concentrate on driving so as to make reactions immediately. In general, when the front car sends out an alarm signal, the driver in the following car can take different measures to avoid collision, including braking and transforming direction etc. [7]. In this paper, the driver is supposed to avoid rear collision by using the parking way.

\subsection{The Analysis of Automotive Braking Process}

Generally speaking, the driver in the following car catches sight of warning signals, recognizes the variation characteristics and then decides to take some measures. This process can be divided into three stages: driver's reaction and braking coordination stage $t_{1}$, deceleration linear growth stage $t_{2}$, and continuous braking stage $t_{3}$ [8]. The curves of braking pedal force $F_{p}$ and braking deceleration $a_{b}$ which vary with time are shown in Figure 1. 


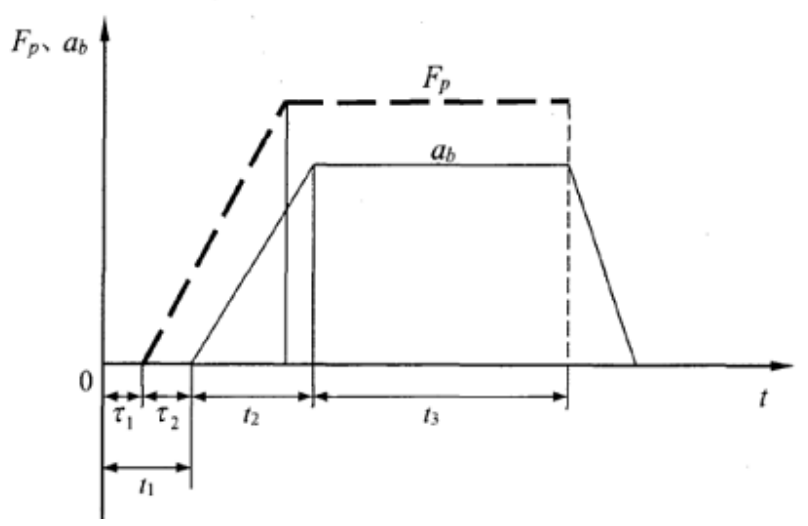

Figure 1. Braking Process of Automobile

Supposing the initial velocity of vehicle is $v_{0}$ and the maximum acceleration is $a_{\max }$. Combined with vehicle's braking kinematics, the following model is established, and the specific derivation can be seen in reference [9].

$$
S=v_{0}\left(t_{1}+\frac{t_{2}}{2}\right)+\frac{v_{0}{ }^{2}}{2 a_{\max }}
$$

\subsection{The Analysis of Automotive Following Process}

An extreme case is assumed: the driver in the following vehicle just realizes the deceleration of the front car and takes emergency measures when the vehicle ahead is braked suddenly. The distance between two vehicles meets stopping distance when both of them stop, which is called the minimum safety distance $D$ [10]. The diagram of safety distance is shown in Figure 2.

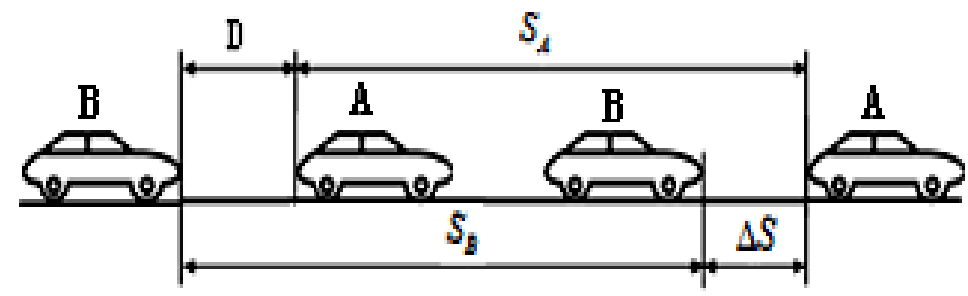

Figure 2. Schematic Diagram of Safety Distance

Through the above analysis, the calculation formula of safety distance can be obtained, shown as follows:

$$
D=S_{B}+\Delta S-S_{A}
$$

Equation (2) shows that the minimum safety distance is related to three elements, which are the braking distance of the front car and the following car, respectively $S_{A}$ and $s_{B}$, and the stopping distance of the two cars $\Delta S$.

\subsection{The Safety Distance Analysis based on the State of the Front Car}

Through the analysis of the front vehicle, driving process can be divided into three situations [11]. Subsequently, the safety distance is to be analyzed in detail. The involving parameters are as follows: $v_{A}$ and $v_{B}$ are the speed of the leading and following vehicle respectively when the preceding car brakes $a_{A}$ and $a_{B}$ are the maximum acceleration of the front and back car respectively. 
3.4.1. The Front Car is Stationary: In this situation, the equivalent of braking distance is zero for front car, so:

$$
D_{1}=v_{B}\left(t_{1}+\frac{t_{2}}{2}\right)+\frac{v_{B}^{2}}{2 a_{B}}+\Delta S
$$

3.4.2. Constant Speed or Accelerated Movement of the Front Car: When the front car is faster than the following car, there is no collision risk. Otherwise, in the whole deceleration process, the most dangerous moment is when the two vehicles' speeds reach equally, Thus, the running distance of these two vehicles from now to that moment should be calculated respectively. Hence:

$$
D_{2}=\frac{\left(v_{B}-v_{A}\right)^{2}}{2 a_{B}}+\left(v_{B}-v_{A}\right) t_{1}+\frac{\left(v_{A}+v_{B}\right) t_{2}}{2}+\Delta S
$$

3.4.3. Decelerated Movement or until Cessation of the Front Car: Whether the front stops earlier or two vehicles stop at the same time, the most dangerous moment will occur at the stop time of the following car, therefore:

$$
D_{3}=v_{B} t_{1}+\frac{\left(v_{B}-v_{A}\right) t_{2}}{2}+\frac{v_{B}{ }^{2}}{2 a_{B}}-\frac{v_{A}{ }^{2}}{2 a_{A}}+\Delta S
$$

\section{The Determination of Parameters in Safety Distance Mathematical Model}

There are many complex variables involved in this model, which possess the characteristics of randomness and fuzziness. Therefore, it is necessary to establish a more accurate, reasonable, and comprehensive mathematical model and apply the fuzzy theory in determining the fuzzy relation among the parameters. Thus, the intelligent system can be in accordance with the practical driving environment of vehicles.

\subsection{The Determination of Velocity and Distance between Two Cars}

The data of two cars' velocity before braking and actual distance between two cars were obtained timely by the vehicle speed sensor and millimeter-wave radar, which were displayed in the man-machine interface in time.

\subsection{The Determination of Parameters of Artificial Selection Unit}

The braking distance of vehicles is affected by different conditions of weather, road, and the corresponding road sticking coefficient. Therefore, the information of weather and road should be selected before the system is started.

4.2.1. The Determination of Weather Information: In this system, four weather condition switches are mounted on the man-machine interface, respectively, "snow", "fog", "rain", and "fine". Defining the four fuzzy subsets of input on weather conditions with fuzzy theory are $\{($ snow $),($ fog $),($ rain $),($ fine $)\}$, their domain used to cover input is $[0,1]$, and the membership function is shown in the formula. 


$$
\begin{aligned}
& \left\{\operatorname{snow}(t q)=\frac{0.18-t q}{0.18}(0 \leq t q \leq 0.18)\right. \\
& f \circ g(t q)=\left\{\begin{array}{l}
\frac{t q-0.15}{0.18}(0.15 \leq t q \leq 0.33) \\
\frac{0.51-t q}{0.18}(0.33 \leq t q \leq 0.51)
\end{array}\right. \\
& \text { rain }(t q)=\left\{\begin{array}{l}
\frac{t q-0.48}{0.18}(0.48 \leq t q \leq 0.66) \\
\frac{0.84-t q}{0.18}(0.66 \leq t q \leq 0.84)
\end{array}\right. \\
& \text { fine }(t q)=\frac{t q-0.82}{0.18}(0.82 \leq t q \leq 1)
\end{aligned}
$$

4.2.2. The Determination of Road Information: Similarly, four road condition switches are mounted on the man-machine interface, respectively, "ice", "soil", "wet", and "dry". Defining the four fuzzy subsets of input on road conditions with fuzzy theory are $\{($ ice $),($ soil $),($ wet $),(d r y)\}$, their domain used to cover input is $[0,1]$, and the membership function is shown in the formula.

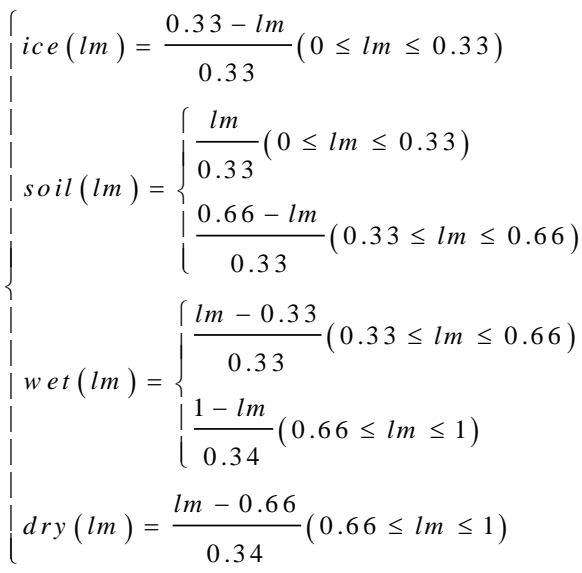

\subsection{The Determination of Time Parameters}

4.3.1. The Determination of $t_{1}: t_{1}$ contains two stages - driver's reaction time $\tau_{1}$ and braking coordination time $\tau_{2}$ [12].Data show that the reaction time of driver is generally $0.4 \square 1.5 \mathrm{~s}$. The vehicles are assumed to use hydraulic brake system whose braking coordination time is $0.3 \mathrm{~s}$ [12], then $t_{1}=\tau_{1}+\tau_{2}=\tau_{1}+0.3(\mathrm{~s}) \cdot \tau_{1}$ is determined directly by the fixed value in the general system. However, the driver's reaction time is closely related to not only the driver's own character but also the weather situation and running speed. Therefore, based on the current driving environment, the fuzzy relationship between reaction time $\tau_{1}$ and weather condition $t q$ as well as speed of the following car $v_{B}$ is established with fuzzy theory, and $\tau_{1}$ is evaluated comprehensively.

Defining the five fuzzy subsets of input on velocity of the following car with fuzzy theory are $\{(d),(z),(p g),(g),(h g)\}$, their domain used to cover input is $[0,200]$, and the membership function is shown in the formula. 


$$
\begin{aligned}
& d\left(v_{B}\right)=\frac{50-v_{B}}{50}\left(0 \leq v_{B} \leq 50\right) \\
& z\left(v_{B}\right)=\left\{\begin{array}{l}
\frac{v_{B}}{50}\left(0 \leq v_{B} \leq 50\right) \\
\frac{100-v_{B}}{50}\left(50 \leq v_{B} \leq 100\right)
\end{array}\right.
\end{aligned}
$$

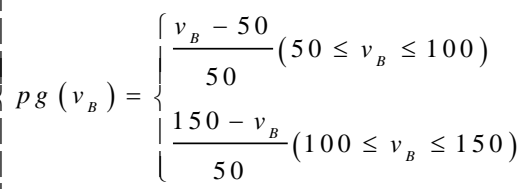

$$
\begin{aligned}
& g\left(v_{B}\right)=\left\{\begin{array}{l}
\frac{v_{B}-100}{50}\left(100 \leq v_{B} \leq 150\right) \\
\frac{200-v_{B}}{50}\left(150 \leq v_{B} \leq 200\right)
\end{array}\right. \\
& h g\left(v_{B}\right)=\frac{v_{B}-150}{50}\left(150 \leq v_{B} \leq 200\right)
\end{aligned}
$$

Defining the three fuzzy subsets of input on reaction time with fuzzy theory are $\{k, z, m\}$, their domain used to cover input is $[0.4,1.5]$, and the membership function is shown in the formula.

$$
\left\{\begin{array}{l}
k\left(\tau_{1}\right)=\frac{0.84-\tau_{1}}{0.44}\left(0.4 \leq \tau_{1} \leq 0.84\right) \\
z\left(\tau_{1}\right)=\left\{\begin{array}{l}
\frac{\tau_{1}-0.51}{0.44}\left(0.51 \leq \tau_{1} \leq 0.95\right) \\
\frac{1.39-\tau_{1}}{0.44}\left(0.95 \leq \tau_{1} \leq 1.39\right)
\end{array}\right. \\
m\left(\tau_{1}\right)=\frac{\tau_{1}-1.06}{0.44}\left(1.06 \leq \tau_{1} \leq 1.5\right)
\end{array}\right.
$$

The fuzzy rule of reaction time $\tau_{1}$ is listed in Table 1 .

Table 1. Fuzzy Rule Table of $\tau_{1}$

\begin{tabular}{ccccc}
\hline Velocity & \multicolumn{4}{c}{ Weather condition $(t q)$} \\
\cline { 2 - 5 }$\left(v_{B}\right)$ & snow & fog & rain & fine \\
$\mathrm{d}$ & $\mathrm{m}$ & $\mathrm{m}$ & $\mathrm{m}$ & $\mathrm{z}$ \\
$\mathrm{z}$ & $\mathrm{m}$ & $\mathrm{m}$ & $\mathrm{z}$ & $\mathrm{k}$ \\
$\mathrm{pg}$ & $\mathrm{m}$ & $\mathrm{z}$ & $\mathrm{z}$ & $\mathrm{k}$ \\
$\mathrm{g}$ & $\mathrm{z}$ & $\mathrm{k}$ & $\mathrm{k}$ & $\mathrm{k}$ \\
$\mathrm{hg}$ & $\mathrm{k}$ & $\mathrm{k}$ & $\mathrm{k}$ & $\mathrm{k}$ \\
\hline
\end{tabular}

4.3.2. The Determination of $t_{2}$ : The growth time of hydraulic braking acceleration is taken as $0.2 \mathrm{~s}$, where: $t_{2}=0.2 \mathrm{~s}[13]$.

\subsection{The Determination of Braking Deceleration}

To simplify the analysis, a hypothesis is presented that two cars' maximum decelerations are the same [14], then $a=a_{A}=a_{B}$. Different road conditions and different running speeds will lead to different ground adhesion coefficients, which result in 
different maximum deceleration. Therefore, based on the current driving environment, the fuzzy relationship between maximum deceleration $a$ and road condition $\mathrm{lm}$ as well as speed of the following $\operatorname{car}_{v_{B}}$ is established with fuzzy theory, and $a$ is evaluated comprehensively.

Defining the seven fuzzy subsets of input on maximum deceleration with fuzzy theory are $\{(h x),(x),(j x),(z),(j d),(d),(h d)\}$, their domain used to cover input is $[0,10]$, and the membership function is shown in the formula.

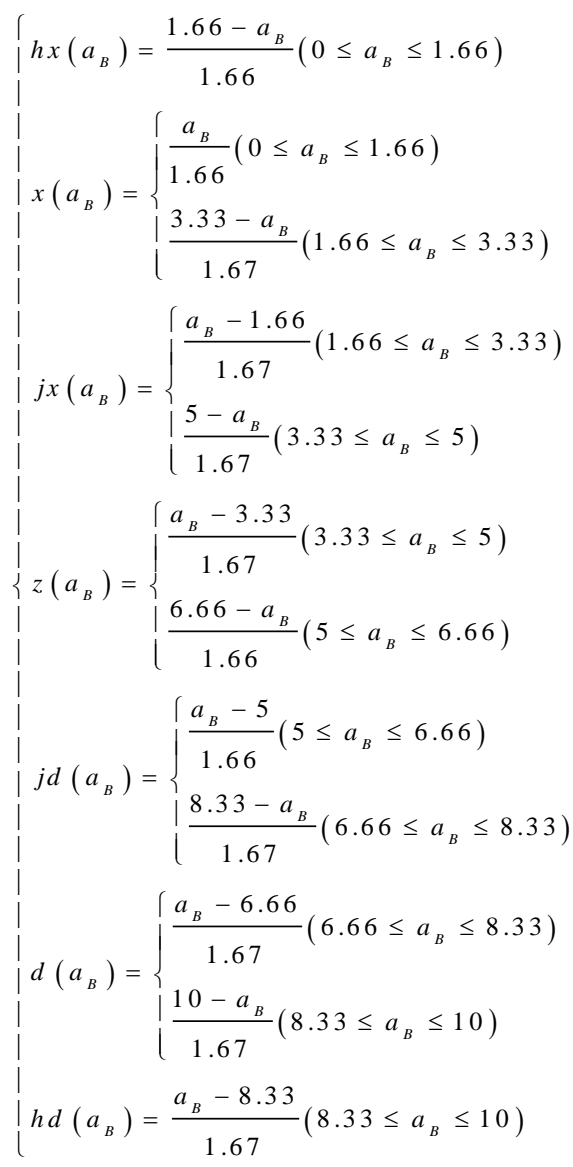

The fuzzy rule of maximum deceleration $a$ is listed in Table 2 .

Table 2. Fuzzy Rule Table of $a$

\begin{tabular}{ccccc}
\hline Velocity & \multicolumn{4}{c}{ Road condition $(l m)$} \\
\cline { 2 - 5 }$\left(v_{B}\right)$ & ice & soil & wet & dry \\
$\mathrm{d}$ & $\mathrm{x}$ & $\mathrm{jd}$ & $\mathrm{d}$ & $\mathrm{hd}$ \\
$\mathrm{z}$ & $\mathrm{x}$ & $\mathrm{z}$ & $\mathrm{jd}$ & $\mathrm{d}$ \\
$\mathrm{pg}$ & $\mathrm{x}$ & $\mathrm{jx}$ & $\mathrm{jd}$ & $\mathrm{d}$ \\
$\mathrm{g}$ & $\mathrm{hx}$ & $\mathrm{jx}$ & $\mathrm{z}$ & $\mathrm{jd}$ \\
$\mathrm{hg}$ & $\mathrm{hx}$ & $\mathrm{x}$ & $\mathrm{jx}$ & $\mathrm{jd}$ \\
\hline
\end{tabular}




\subsection{The Determination of Safety Stopping Distance}

The value of safety stopping distance $\Delta S$ reflects the performance of warning system, that is the larger $\Delta S$ is, the higher rate the false alarm achieves, and vice versa [15]. $\Delta S$ is usually taken as $2 \square 5 m$, and the distance is chosen as $3 m$ [11].

\section{Simulation Results and Analysis}

In order to make the calculation simple and achieve clear results, the front car is supposed to be stationary in the simulation experiment, that is $v_{A}=0$.

The value of parameters in most of current anti-collision systems are set as fixed, according to reference [5], where: $t_{1}=1.3 \mathrm{~s}, t_{2}=0.2 \mathrm{~s}$; the value of acceleration on dry condition is $a=8.5 \mathrm{~m} / \mathrm{s}^{2}$, and the value decreases to $a=7 \mathrm{~m} / \mathrm{s}^{2}$ on wet road, and to $a=6 \mathrm{~m} / \mathrm{s}^{2}$ on soil road, and to $a=1.5 \mathrm{~m} / \mathrm{s}^{2}$ on ice road.

A simulation system based on safety distance model is established by means of MATLAB and the simulation experiment is carried out. The results of simulation and calculation are shown in Table 3.

Table 3. Safety Distance in $v_{A}=0$ and Different Weather, Road, Speed Conditions

\begin{tabular}{lllllllllll}
\hline$v_{B}(\mathrm{~km} / \mathrm{h})$ & & 20 & 30 & 40 & 50 & 60 & 70 & 80 & 90 & 100 \\
\hline fine dry & Simulation & 11.9 & 17.1 & 21.7 & 27.4 & 35.3 & 44.3 & 53.9 & 64 & 75.1 \\
& Calculation & 12.3 & 18.6 & 25.8 & 33.8 & 42.7 & 52.5 & 63.2 & 74.8 & 87.3 \\
fog dry & Simulation & 14.5 & 21.6 & 30 & 39.3 & 47.7 & 55 & 64.2 & 75 & 87 \\
& Calculation & 12.3 & 18.6 & 25.8 & 33.8 & 42.7 & 52.5 & 63.2 & 74.8 & 87.3 \\
rain wet & Simulation & 13.3 & 19.6 & 27.2 & 36.1 & 46.2 & 57.5 & 69.9 & 83.4 & 98.1 \\
& Calculation & 13 & 19.6 & 27.4 & 36.2 & 46.2 & 57.2 & 69.4 & 82.6 & 97 \\
fine soil & Simulation & 12.7 & 19.2 & 26.2 & 35.1 & 47.5 & 64.5 & 87.5 & 115.6 & 145.5 \\
& Calculation & 13.3 & 20.5 & 28.8 & 38.5 & 49.5 & 61.7 & 75.3 & 90.1 & 106.2 \\
snow ice & Simulation & 21.8 & 38 & 59 & 84.4 & 114.2 & 148.2 & 187 & 231 & 279.4 \\
& Calculation & 21.1 & 37.8 & 59.7 & 86.7 & 118.9 & 156.3 & 198.7 & 246.3 & 299.1 \\
\hline
\end{tabular}

By analyzing the data in Table 3, we can put forward some findings as following:

(1) Under the same condition, the safety distance gradually increases with the increasing of car's speed, which indicates the method is reliable.

(2) Under the same road condition and at the same speed, weather also has some influence on the safety distance, which results from the fact that weather sometimes may have influence on people's moods.

(3) Under the same conditions of weather, road, and speed, the result of safety distance of simulation is relatively smaller than that in calculation, which can dramatically reduce the false alarm.

In summary, the factors which can affect safety distance include not only the road and weather conditions but also the driver and vehicle system. In the mean time, there exist many uncertainties among these factors. Thus, the accuracy of man-machine interaction can be greatly improved when variables are determined by means of fuzzy theory, which is of great significance for the system. 


\section{Conclusion}

The pro-active head restraint is an essential safety protection device to passenger's whiplash injury, and the safety distance mathematical model is a core part of the rear-end collision avoidance system. At present, most models are set with fixed parameters, which have caused false alarm problems frequently. Based on the above analysis, this parer presents the safety distance model established on the traditional dynamics. The system fully takes into account the the driver's characteristics, weather conditions, road situations and vehicle's characteristics with fuzzy theory so as to obtain the accurate and real-time safety distance model. The experiment has revealed that the mathematical model is more applicable to the actual traffic environment, and it's of great significance for the development of anti-collision system, which promotes the application of pro-active head restraint. However, the paper does not take the vehicle lane changing into consideration, the model still needs further study.

\section{Acknowledgements}

This paper is based on research that was sponsored by the Entrepreneurship Program Foundation of Wuxi (No.2011091011).

\section{References}

[1] ITARDA Report of Vehicle Safety Measures Study Based on Traffic Accidents Analysis for 2007 FY, (2007).

[2] NHTSA, Traffic safety facts 2009. U.S. Department of transportation, National Highway Traffic Safety Administration, DOT HS 811 402, (2009).

[3] J. Lin, H. L. Du and Q. Zhou, "Study on control strategy of active headrest in rear-end collision", Automotive technology, (2006), pp. 52-88.

[4] R. Zheng, "Design and realization of millimeter wave radar for collision avoidance", Jiangsu university, Nanjing, (2010).

[5] G. W. Chen, D. B. Hou and X. X. Li, "The practical safety distance calculation model in highway", Human ergonomics, vol. 7, no. 1, (2001), pp. 41-44.

[6] J. Ma, "Analysis and research of safety distance in highway", Journal of Xian Jiaotong university, vol. 18, no. 4, (1998), pp. 90-94.

[7] Y. Zhong and J. F. Yao, "Discussion on the critical safety distance of vehicle", Journal of Hunan university, vol. 28, no. 6, (2001), pp. 54-58.

[8] M. Z. Deng and X. F. Ruan, "Research on the smallest safety driving distance mathematical model in highway", Water transportation of China, vol. 66, (2006), pp. 109-110.

[9] Z. S. Yu, "Automobile theory", Machinery Industry Press, Beijing, (2011).

[10] J. Y. Lian and X. Y. Hua, "Study on mathematical model of rear-end collision avoidance", Journal of Chinese highway, vol. 18, no. 3, (2005), pp. 123-126.

[11] J. L. Ma, "Study on automobile anti-collision system", North Western polytechnical university, Xian, (2007).

[12] Y. S. Sheng and L. Ma, "Research on anti-collision barking strategy", Mechanical engineering of China, vol. 4, no. 3, (2007), pp. 66-71.

[13] H. X. Zhang, "Dynamic system of vehicle", Tongji university Press,Shanghai, (2002).

[14] X. X. Li, B. C. Li and D. B. Hou, "Study on the development of automobile rear collision warning system", Journal of Chinese highway, vol. 14, no. 3, (2001), pp. 93-95.

[15] J. Q. Liang, "Research on the development of anti-collision warning system for full 360 degree", Changan university, (2008). 


\section{Authors}

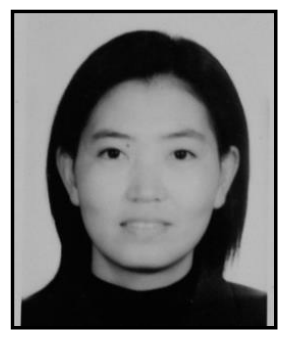

Xiaoqin Yin, she received the B.S. degree in mechanical engineering from Jiangsu University, Zhenjiang, China, in 1993. She received the M.S. and Ph.D. degree in Mechanical Engineering from Jiangsu University in 2005. She is currently an associate professor in College of Mechanical Engineering of Jiangsu University. Her current research interests include the theory of parallel robot, mechanism and mechanical vibration.

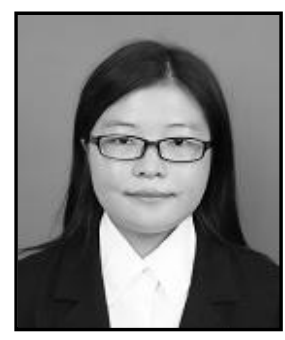

Mingxia Wang, she received the B.S. degree in mechanical engineering from Jiangsu Institute of Technology, Changzhou, China, in 2012. Now, she is working toward the M.S degree in College of Mechanical Engineering of Jiangsu University, Zhenjiang, China. Her current research interest is in design and research of a new automobile pro-active head restraint of electromagnetic drive. 